Acta Crystallographica Section E

Structure Reports

Online

ISSN 1600-5368

\section{2-(8-Bromoimidazo[1,2-a]pyridin-2-yl)- $N^{\prime}$-[(E)-4-diethylamino-2-hydroxybenzyl- idene]acetohydrazide dihydrate}

\author{
Hoong-Kun Fun, ${ }^{a *} \mp$ Wan-Sin Loh, ${ }^{a} \S$ Seema Shenvi, ${ }^{b}$ \\ Arun M. Isloor ${ }^{b}$ and Gurumurthy Hegde ${ }^{c}$
}

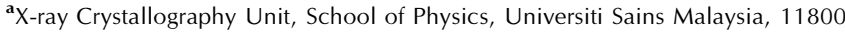
USM, Penang, Malaysia, ${ }^{\mathbf{b}}$ Organic Electronics Division, Department of Chemistry, National Institute of Technology - Karnataka, Surathkal, Mangalore 575 025, India and ${ }^{\mathrm{C}}$ Faculty of Industrial Science and Technology, Universiti Malaysia Pahang, Lebuhraya Tun Razak, 26300 Gambang, Kuantan, Pahang Darul Makmur, Malaysia Correspondence e-mail: hkfun@usm.my

Received 15 February 2012; accepted 15 February 2012

Key indicators: single-crystal X-ray study; $T=100 \mathrm{~K}$; mean $\sigma(\mathrm{C}-\mathrm{C})=0.002 \AA$; $R$ factor $=0.027 ; w R$ factor $=0.077 ;$ data-to-parameter ratio $=21.9$.

In the title compound, $\mathrm{C}_{20} \mathrm{H}_{22} \mathrm{BrN}_{5} \mathrm{O}_{2} \cdot 2 \mathrm{H}_{2} \mathrm{O}$, the Schiff base molecule exists in an $E$ conformation with respect to the acyclic $\mathrm{C}=\mathrm{N}$ bond. An $S(6)$ ring motif is formed via an intramolecular $\mathrm{O}-\mathrm{H} \cdots \mathrm{N}$ hydrogen bond. The dihedral angle between the imidazo[1,2- $a$ ]pyridine system and the benzene ring is $84.62(5)^{\circ}$. In the crystal, $\mathrm{N}-\mathrm{H} \cdots \mathrm{O}, \mathrm{O}-\mathrm{H} \cdots \mathrm{O}, \mathrm{O}-$ $\mathrm{H} \cdots \mathrm{N}, \mathrm{C}-\mathrm{H} \cdots \mathrm{O}$ and $\mathrm{C}-\mathrm{H} \cdots \mathrm{Br}$ hydrogen bonds link the molecules into a three-dimensional network. The crystal packing is further stabilized by $\mathrm{C}-\mathrm{H} \cdots \pi$ and $\pi-\pi$ interactions [centroid-centroid distance $=3.5365(7) \AA$ ] .

\section{Related literature}

For background to and applications of hydrazones, see: Seleem et al. (2011); Rollas \& Küçükgüzel (2007). For background to and applications of imidazopyridine, see: Ertepinarl et al. (1995); Liang et al. (2007); Hamdouchi et al. (1999); Gudmundsson \& Johns (2007); Biftu et al. (2006); Fisher \& Lusi (1972); Bochis et al. (1981). For hydrogen-bond motifs, see: Bernstein et al. (1995). For the stability of the temperature controller used for the data collection, see: Cosier \& Glazer (1986).

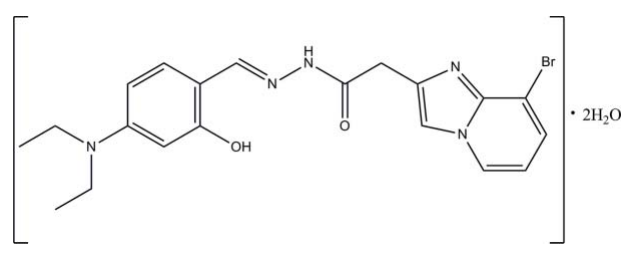

\section{Experimental}

Crystal data

$\mathrm{C}_{20} \mathrm{H}_{22} \mathrm{BrN}_{5} \mathrm{O}_{2} \cdot 2 \mathrm{H}_{2} \mathrm{O}$

$M_{r}=480.37$

Triclinic, $P \overline{1}$

$a=8.4370$ (4) ^

$b=10.6711(5) \AA$

$c=11.7559(5) \AA$

$\alpha=92.914$ (1)

$\beta=96.949$ (1)

$\gamma=93.978(1)^{\circ}$

$V=1046.23(8) \AA^{3}$

$Z=2$

Mo $K \alpha$ radiation

$\mu=2.00 \mathrm{~mm}^{-1}$

$T=100 \mathrm{~K}$

$0.37 \times 0.20 \times 0.07 \mathrm{~mm}$

\section{Data collection}

Bruker SMART APEXII CCD diffractometer

Absorption correction: multi-scan (SADABS; Bruker, 2009)

$T_{\min }=0.529, T_{\max }=0.869$

Refinement

$R\left[F^{2}>2 \sigma\left(F^{2}\right)\right]=0.027$

$w R\left(F^{2}\right)=0.077$

$S=1.05$

6491 reflections

297 parameters

22937 measured reflections 6491 independent reflections 5841 reflections with $I>2 \sigma(I)$ $R_{\text {int }}=0.023$

$\mathrm{H}$ atoms treated by a mixture of independent and constrained refinement

$\Delta \rho_{\max }=0.63{\mathrm{e} \AA^{-3}}^{-3}$

$\Delta \rho_{\min }=-0.24{\mathrm{e} \AA^{-3}}^{-3}$

Table 1

Hydrogen-bond geometry $\left(\AA{ }^{\circ}\right)$.

$C g 1$ is the centroid of the $\mathrm{N} 1 / \mathrm{C} 1 / \mathrm{N} 2 / \mathrm{C} 6 / \mathrm{C} 7$ ring and $C g 3$ is the centroid of the C11-C16 ring.

\begin{tabular}{lllll}
\hline$D-\mathrm{H} \cdots A$ & $D-\mathrm{H}$ & $\mathrm{H} \cdots A$ & $D \cdots A$ & $D-\mathrm{H} \cdots A$ \\
\hline $\mathrm{N} 3-\mathrm{H} 1 \mathrm{~N} 3 \cdots \mathrm{O} 2 W^{\mathrm{i}}$ & $0.894(17)$ & $1.908(17)$ & $2.7956(15)$ & $171.7(17)$ \\
$\mathrm{O} 2-\mathrm{H} 1 \mathrm{O} 2 \cdots \mathrm{O} 1 W^{\mathrm{ii}}$ & $0.87(3)$ & $2.42(3)$ & $2.9423(15)$ & $119(2)$ \\
$\mathrm{O} 2-\mathrm{H} 1 \mathrm{O} 2 \cdots \mathrm{N} 4$ & $0.87(3)$ & $1.99(3)$ & $2.7181(16)$ & $142(2)$ \\
$\mathrm{O} 1 W-\mathrm{H} 1 W 1 \cdots \mathrm{N} 1$ & $0.86(2)$ & $1.98(2)$ & $2.8315(14)$ & $176(2)$ \\
$\mathrm{O} 1 W-\mathrm{H} 2 W 1 \cdots \mathrm{O} 1^{\mathrm{iii}}$ & $0.85(3)$ & $1.92(2)$ & $2.7361(14)$ & $162(2)$ \\
$\mathrm{O} 2 W-\mathrm{H} 1 W 2 \cdots \mathrm{O} 1 W^{\mathrm{i}}$ & $0.80(2)$ & $2.08(2)$ & $2.8311(15)$ & $157(2)$ \\
$\mathrm{O} 2 W-\mathrm{H} 2 W 2 \cdots \mathrm{O} 1 W^{2}$ & $0.86(2)$ & $1.87(2)$ & $2.7245(15)$ & $172.9(17)$ \\
$\mathrm{C} 5-\mathrm{H} 5 A \cdots \mathrm{O} 1^{\text {iv }}$ & 0.93 & 2.50 & $3.3121(17)$ & 146 \\
$\mathrm{C} 10-\mathrm{H} 10 A \cdots \mathrm{O} 2 W^{\mathrm{i}}$ & 0.93 & 2.54 & $3.3256(17)$ & 142 \\
$\mathrm{C} 17-\mathrm{H} 17 B \cdots \mathrm{Br} 1^{\mathrm{v}}$ & 0.97 & 2.85 & $3.6569(15)$ & 142 \\
$\mathrm{C} 3-\mathrm{H} 3 A \cdots C g 3^{\text {vi }}$ & 0.93 & 2.61 & $3.4734(15)$ & 154 \\
$\mathrm{C} 17-\mathrm{H} 17 A \cdots C g 1^{\text {vii }}$ & 0.97 & 2.70 & $3.5863(15)$ & 152 \\
\hline
\end{tabular}

Symmetry codes: (i) $-x+1,-y,-z+1$; (ii) $x-1, y, z$; (iii) $x+1, y, z$; (iv)

$-x,-y+1,-z+1 ;(\mathrm{v})-x+1,-y,-z+2 ;$ (vi) $x+1, y+1, z$; (vii) $-x,-y,-z+2$.

Data collection: APEX2 (Bruker, 2009); cell refinement: SAINT (Bruker, 2009); data reduction: $S A I N T$; $\operatorname{program}(\mathrm{s})$ used to solve structure: SHELXTL (Sheldrick, 2008); program(s) used to refine structure: SHELXTL; molecular graphics: SHELXTL; software used to prepare material for publication: SHELXTL and PLATON (Spek, 2009).

HKF and WSL thank Universiti Sains Malaysia (USM) for Research University Grant No. 1001/PFIZIK/811160. WSL also thanks the Malaysian Government and USM for the award of the post of Research Officer under Research University Grant No. 1001/PFIZIK/811160. AMI thanks the Department of Atomic Energy, Board for Research in Nuclear Sciences, Government of India, for a Young Scientist award.

Supplementary data and figures for this paper are available from the IUCr electronic archives (Reference: BQ2340). 


\section{References}

Bernstein, J., Davis, R. E., Shimoni, L. \& Chang, N.-L. (1995). Angew. Chem. Int. Ed. Engl. 34, 1555-1573.

Biftu, T. et al. (2006). Bioorg. Med. Chem. Lett. 16, 2479-2483.

Bochis, R. J., Olen, L. E., Waksmunski, F. S., Mrozik, H., Eskola, P., Kulsa, P., Wilks, G., Taylor, J. E., Egerton, J. R., Ostlind, D. A. \& Olson, G. (1981). J. Med. Chem. 24, 1518-1521.

Bruker (2009). APEX2, SAINT and SADABS. Bruker AXS Inc., Madison, Wisconsin, USA.

Cosier, J. \& Glazer, A. M. (1986). J. Appl. Cryst. 19, 105-107.

Ertepinarl, H., Gök, Y., Geban, Ö. \& Özden, S. (1995). Eur. J. Med. Chem. 30, 171-175.
Fisher, M. H. \& Lusi, A. (1972). J. Med. Chem. 15, 982-985.

Gudmundsson, K. S. \& Johns, B. A. (2007). Bioorg. Med. Chem. Lett. 17, 27352739.

Hamdouchi, C., Ezquerra, J., Vega, J. A., Vaquero, J. J., Alvarez-Builla, J. \& Heinz, B. A. (1999). Bioorg. Med. Chem. Lett. 9, 1391-1394.

Liang, G.-B., Qian, X., Feng, D., Fisher, M., Brown, C. M., Gurnett, A., Leavitt, P. S., Liberator, P. A., Misura, A. S., Tamas, T., Schmatz, D. M., Wyvratt, M. \& Biftu, T. (2007). Bioorg. Med. Chem. Lett. 17, 3558-3561.

Rollas, S. \& Küçükgüzel, Ş. G. (2007). Molecules, 12, 1910-1939.

Seleem, H. S., El-Inany, G. A., El-Shetary, B. A. \& Mousa, M. A. (2011). Chem. Cent. J. doi:10.1186/1752-153X-5-2.

Sheldrick, G. M. (2008). Acta Cryst. A64, 112-122.

Spek, A. L. (2009). Acta Cryst. D65, 148-155. 


\section{supporting information}

Acta Cryst. (2012). E68, o816-o817 [doi:10.1107/S160053681200685X]

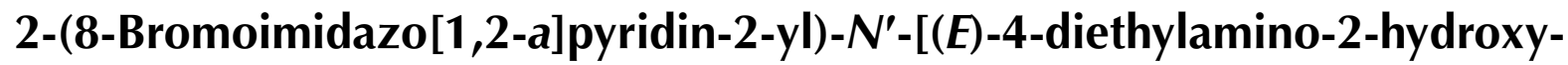 benzylidene]acetohydrazide dihydrate}

\section{Hoong-Kun Fun, Wan-Sin Loh, Seema Shenvi, Arun M. Isloor and Gurumurthy Hegde}

\section{S1. Comment}

Hydrazones constitute an important class of biologically active drug molecules (Seleem et al., 2011) which has attracted attention of medicinal chemists due to their wide range of pharmacological properties. These compounds are being synthesized as drugs by many researchers in order to combat diseases with minimal toxicity and maximal effects. A number of hydrazone derivatives have been reported to exert notably antimicrobial, antihypertensive, anticonvulsant, analgesic, anti-inflammatory, antituberculosis, antitumoral, antiproliferative and antimalarial activities (Rollas \& Küçükgüzel, 2007). Imidazopyridine is the fundamental heterocyclic component of principal anthelmintic drugs. In addition, the imidazopyridine ring possesses many anti-infective properties including antibacterial (Ertepinarl et al., 1995; Liang et al., 2007), antiviral (Hamdouchi et al., 1999; Gudmundsson \& Johns, 2007), antiprotozoal (Biftu et al., 2006) and especially anthelmintic (Fisher \& Lusi, 1972; Bochis et al., 1981) activities. Therefore, the imidazopyridine ring could replace the benzimidazole ring in the design and the development of new anthelmintic agents. In view of its biological importance, we hereby report the crystal structure of (I).

The title compound (Fig. 1) consists of one 2-(8-bromoimidazo[1,2-a]pyridin-3-yl)- $N^{\prime}-\{(E)$ - [4-(diethylamino)-2-hydroxyphenyl]methylidene acetohydrazide molecule and two water molecules. The Schiff base molecule exists in an $E$ configuration with respect to the acyclic $\mathrm{C}=\mathrm{N}$ bond. An $S(6)$ ring motif (Bernstein et al., 1995) is formed via the intramolecular $\mathrm{O} 2-\mathrm{H} 1 \mathrm{O} 2 \cdots \mathrm{N} 4$ hydrogen bond. The dihedral angle between the imidazo[1,2-a]pyridine (C1$\mathrm{C} 5 / \mathrm{N} 2 / \mathrm{C} 6 / \mathrm{C} 7 / \mathrm{N} 1)$ and the benzene $(\mathrm{C} 11-\mathrm{C} 16)$ rings is $84.62(5)^{\circ}$.

In the crystal packing (Fig. 2), intermolecular $\mathrm{N} 3-\mathrm{H} 1 \mathrm{~N} 3 \cdots \mathrm{O} 2 \mathrm{~W}, \mathrm{O} 2-\mathrm{H} 1 \mathrm{O} 2 \cdots \mathrm{O} 1 \mathrm{~W}, \mathrm{O} 2-\mathrm{H} 1 \mathrm{O} 2 \cdots \mathrm{N} 4, \mathrm{O} 1 \mathrm{~W}-$ $\mathrm{H} 1 \mathrm{~W} 1 \cdots \mathrm{N} 1, \mathrm{O} 1 \mathrm{~W}-\mathrm{H} 2 \mathrm{~W} 1 \cdots \mathrm{O} 1, \mathrm{O} 2 \mathrm{~W}-\mathrm{H} 1 \mathrm{~W} 2 \cdots \mathrm{O} 1 \mathrm{~W}, \mathrm{O} 2 \mathrm{~W}-\mathrm{H} 2 \mathrm{~W} 2 \cdots \mathrm{O} 1 \mathrm{~W}, \mathrm{C} 5-\mathrm{H} 5 \mathrm{~A} \cdots \mathrm{O} 1, \mathrm{C} 10-\mathrm{H} 10 \mathrm{~A} \cdots \mathrm{O} 2 \mathrm{~W}$ and $\mathrm{C} 17-\mathrm{H} 17 \mathrm{~B} \cdots \mathrm{Br} 1$ hydrogen bonds link the molecules into a three-dimensional network. The crystal packing is further stabilized by $\mathrm{C}-\mathrm{H} \cdots \pi$ interactions, involving the $1 \mathrm{H}$-imidazole $(\mathrm{N} 1 / \mathrm{C} 1 / \mathrm{N} 2 / \mathrm{C} 6 / \mathrm{C} 7 ; \mathrm{Cg} 1$; Table 1$)$ and benzene $(\mathrm{Cg} 3$; Table 1) rings. Weak $\pi-\pi$ interactions were observed involving $1 H$-imidazole, pyridine (N2/C1-C5; $\mathrm{Cg} 2)$ and benzene rings. $[C g 1 \cdots C g 1=3.5365$ (7) $\AA$; symmetry code: $1-x, 1-y, 1-z ; C g 1 \cdots C g 2=3.6210$ (7) $\AA$; symmetry code: $1-x, 1$ $y, 1-z ; C g 3 \cdots C g 3=3.6253(8) \AA$; symmetry code: $-x,-y, 2-z]$.

\section{S2. Experimental}

The mixture of 2-(8-bromoimidazo[1,2-a]pyridine-3-yl)acetohydrazide (200 mg, $0.00074 \mathrm{~mol}$ ), 4-(diethylamino)-2-hydroxy benzaldehyde $(143.6 \mathrm{mg}, 0.00074 \mathrm{~mol})$ and a catalytic quantity of acetic acid $(0.1 \mathrm{ml})$ and ethanol (10 ml) was stirred overnight at $90^{\circ} \mathrm{C}$. On cooling, orange plate-shaped crystals of the product begins to separate. It was collected by filtration and recrystallized from ethanol. Yield: $307.7 \mathrm{mg}$, 93.2\%. m.p. 401-402 K. 


\section{S3. Refinement}

$\mathrm{O}$ - and $\mathrm{N}$-bound $\mathrm{H}$ atoms were located from the difference Fourier map and were refined freely $[\mathrm{O}-\mathrm{H}=0.80$ (3) to 0.87 (3) $\AA ; \mathrm{N}-\mathrm{H}=0.896$ (19) $\AA$ ]. The remaining $\mathrm{H}$ atoms were positioned geometrically and refined with a riding model with $U_{\text {iso }}(\mathrm{H})=1.2$ or $1.5 U_{\text {eq }}(\mathrm{C})[\mathrm{C}-\mathrm{H}=0.93$ to $0.97 \AA]$. A rotating group model was applied to the methyl groups. Three outliners were omitted in the final refinement, $230, \overline{54} 1$ and $3 \overline{6} 5$.

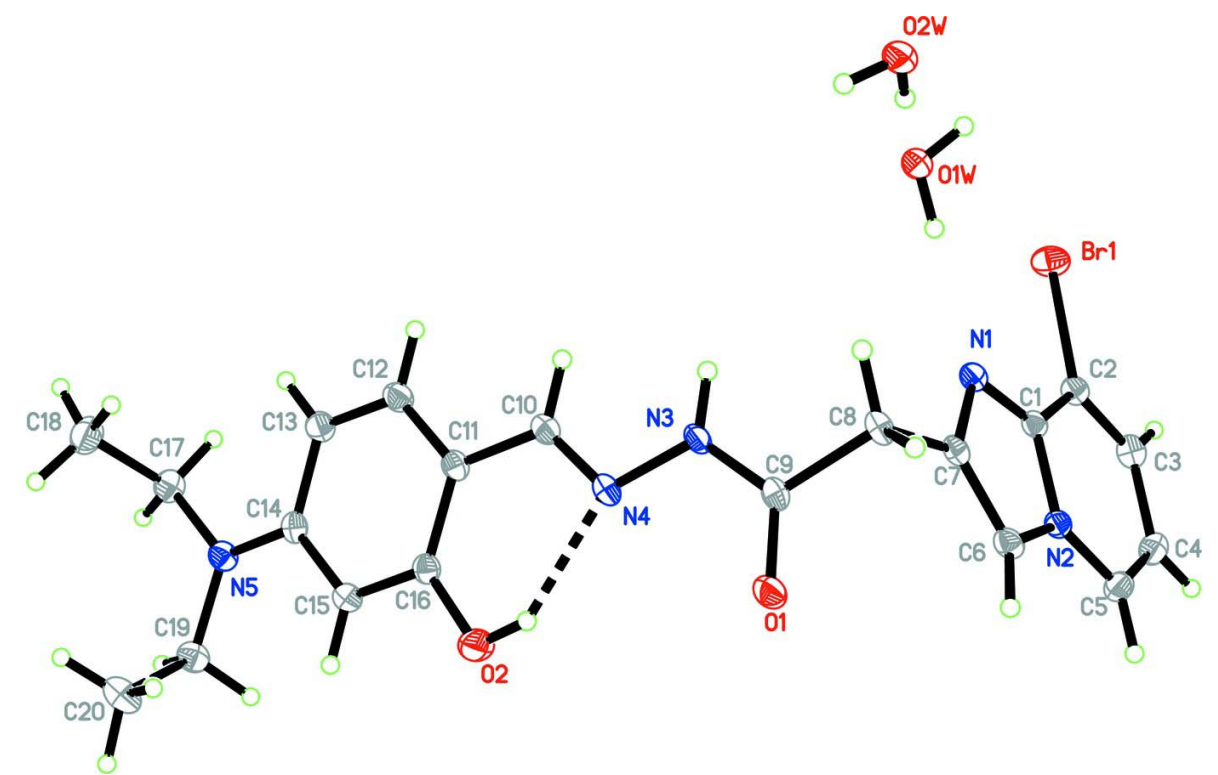

\section{Figure 1}

The molecular structure of the title compound, showing 50\% probability displacement ellipsoids. Dashed line indicates the intramolecular hydrogen bond. 


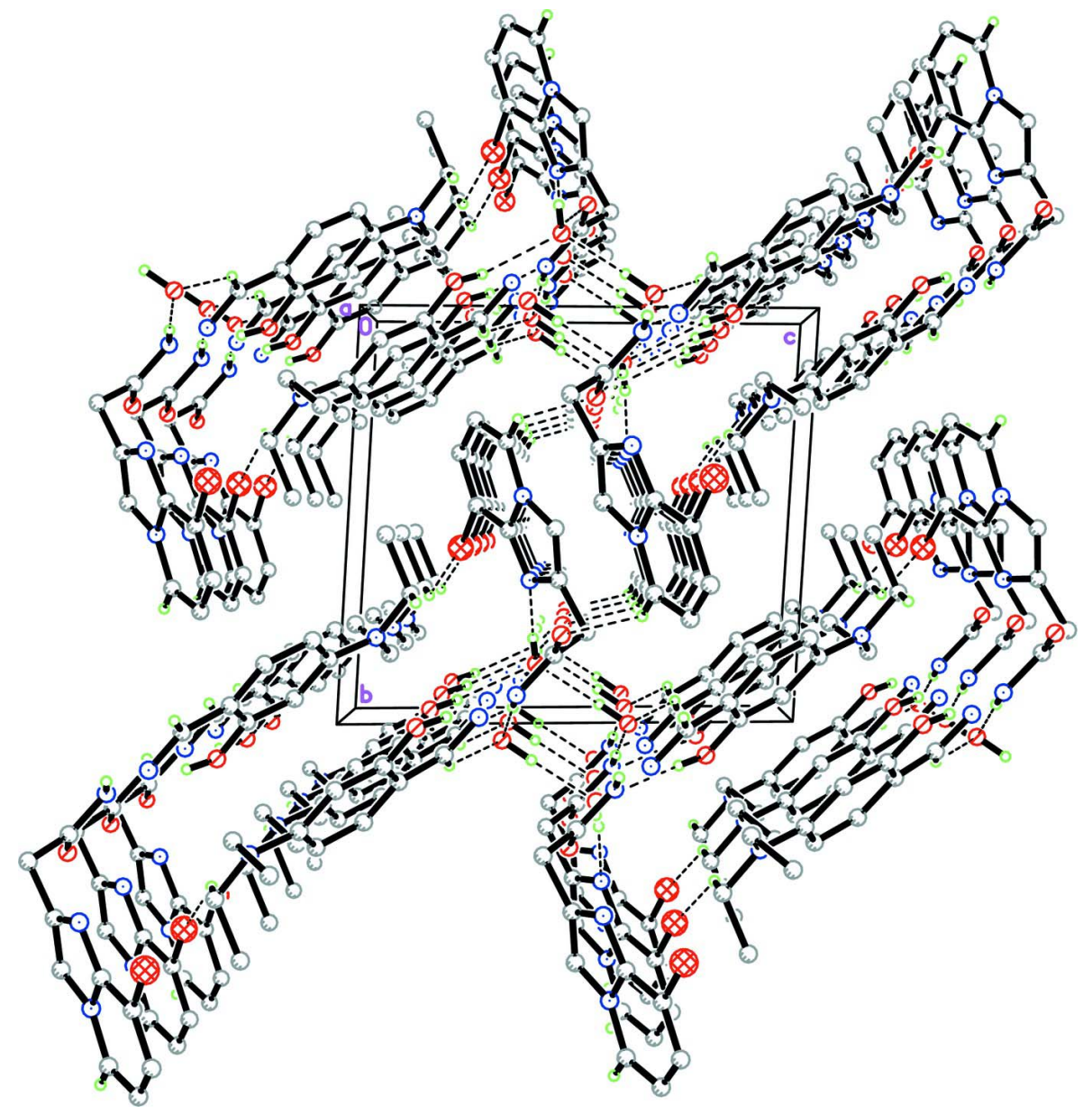

Figure 2

The crystal packing of the title compound, viewed along the $a$ axis, showing the three-dimensional network. H atoms not involved in the intermolecular interactions (dashed lines) have been omitted for clarity.

2-(8-Bromoimidazo[1,2-a]pyridin-2-yl)- $N^{\prime}-[(E)$ - 4-diethylamino-2-hydroxybenzylidene]acetohydrazide dihydrate

Crystal data

$\mathrm{C}_{20} \mathrm{H}_{22} \mathrm{BrN}_{5} \mathrm{O}_{2} \cdot 2 \mathrm{H}_{2} \mathrm{O}$

$M_{r}=480.37$

Triclinic, $P \overline{1}$

Hall symbol: -P 1

$a=8.4370(4) \AA$

$b=10.6711(5) \AA$

$c=11.7559(5) \AA$

$\alpha=92.914(1)^{\circ}$

$\beta=96.949(1)^{\circ}$

$\gamma=93.978(1)^{\circ}$

$V=1046.23(8) \AA^{3}$

Data collection

Bruker SMART APEXII CCD

diffractometer

Radiation source: fine-focus sealed tube
$Z=2$

$F(000)=496$

$D_{\mathrm{x}}=1.525 \mathrm{Mg} \mathrm{m}^{-3}$

Mo $K \alpha$ radiation, $\lambda=0.71073 \AA$

Cell parameters from 9983 reflections

$\theta=2.4-30.9^{\circ}$

$\mu=2.00 \mathrm{~mm}^{-1}$

$T=100 \mathrm{~K}$

Plate, orange

$0.37 \times 0.20 \times 0.07 \mathrm{~mm}$

Graphite monochromator

$\varphi$ and $\omega$ scans 
Absorption correction: multi-scan

(SADABS; Bruker, 2009)

$T_{\min }=0.529, T_{\max }=0.869$

22937 measured reflections

6491 independent reflections

5841 reflections with $I>2 \sigma(I)$

\section{Refinement}

Refinement on $F^{2}$

Least-squares matrix: full

$R\left[F^{2}>2 \sigma\left(F^{2}\right)\right]=0.027$

$w R\left(F^{2}\right)=0.077$

$S=1.05$

6491 reflections

297 parameters

0 restraints

Primary atom site location: structure-invariant

direct methods

$$
\begin{aligned}
& R_{\text {int }}=0.023 \\
& \theta_{\text {max }}=30.9^{\circ}, \theta_{\text {min }}=1.8^{\circ} \\
& h=-12 \rightarrow 12 \\
& k=-15 \rightarrow 15 \\
& l=-16 \rightarrow 16
\end{aligned}
$$

Secondary atom site location: difference Fourier map

Hydrogen site location: inferred from neighbouring sites

$\mathrm{H}$ atoms treated by a mixture of independent and constrained refinement

$w=1 /\left[\sigma^{2}\left(F_{\mathrm{o}}^{2}\right)+(0.0455 P)^{2}+0.2952 P\right]$ where $P=\left(F_{\mathrm{o}}{ }^{2}+2 F_{\mathrm{c}}{ }^{2}\right) / 3$

$(\Delta / \sigma)_{\max }=0.002$

$\Delta \rho_{\max }=0.63$ e $\AA^{-3}$

$\Delta \rho_{\min }=-0.24$ e $\AA^{-3}$

\section{Special details}

Experimental. The crystal was placed in the cold stream of an Oxford Cryosystems Cobra open-flow nitrogen cryostat (Cosier \& Glazer, 1986) operating at 100.0 (1) K.

Geometry. All e.s.d.'s (except the e.s.d. in the dihedral angle between two 1.s. planes) are estimated using the full covariance matrix. The cell e.s.d.'s are taken into account individually in the estimation of e.s.d.'s in distances, angles and torsion angles; correlations between e.s.d.'s in cell parameters are only used when they are defined by crystal symmetry. An approximate (isotropic) treatment of cell e.s.d.'s is used for estimating e.s.d.'s involving l.s. planes.

Refinement. Refinement of $F^{2}$ against ALL reflections. The weighted $R$-factor $w R$ and goodness of fit $S$ are based on $F^{2}$, conventional $R$-factors $R$ are based on $F$, with $F$ set to zero for negative $F^{2}$. The threshold expression of $F^{2}>\sigma\left(F^{2}\right)$ is used only for calculating $R$-factors $(\mathrm{gt})$ etc. and is not relevant to the choice of reflections for refinement. $R$-factors based on $F^{2}$ are statistically about twice as large as those based on $F$, and $R$-factors based on ALL data will be even larger.

Fractional atomic coordinates and isotropic or equivalent isotropic displacement parameters $\left(\AA^{2}\right)$

\begin{tabular}{lllll}
\hline & $x$ & $y$ & $z$ & $U_{\text {iso }} * / U_{\text {eq }}$ \\
\hline Br1 & $0.789019(15)$ & $0.424051(13)$ & $0.748329(12)$ & $0.02141(5)$ \\
O1 & $-0.05933(11)$ & $0.23815(10)$ & $0.52050(9)$ & $0.02047(19)$ \\
O2 & $-0.26058(13)$ & $0.04472(10)$ & $0.79255(9)$ & $0.0227(2)$ \\
N1 & $0.44340(12)$ & $0.35221(10)$ & $0.59413(9)$ & $0.01348(19)$ \\
N2 & $0.35279(12)$ & $0.54369(10)$ & $0.61740(9)$ & $0.01344(19)$ \\
N3 & $0.10753(13)$ & $0.09994(10)$ & $0.60467(9)$ & $0.01444(19)$ \\
N4 & $-0.00110(13)$ & $0.05569(10)$ & $0.67606(9)$ & $0.0150(2)$ \\
N5 & $-0.25929(13)$ & $-0.22618(11)$ & $1.10154(9)$ & $0.0164(2)$ \\
C1 & $0.47756(14)$ & $0.46769(11)$ & $0.64404(10)$ & $0.0127(2)$ \\
C2 & $0.61422(15)$ & $0.52232(12)$ & $0.71616(11)$ & $0.0147(2)$ \\
C3 & $0.62011(16)$ & $0.64472(13)$ & $0.75761(11)$ & $0.0174(2)$ \\
H3A & 0.7093 & 0.6799 & 0.8059 & $0.021^{*}$ \\
C4 & $0.48898(17)$ & $0.71799(12)$ & $0.72639(11)$ & $0.0182(2)$ \\
H4A & 0.4935 & 0.8015 & 0.7542 & $0.022^{*}$ \\
C5 & $0.35760(16)$ & $0.66812(12)$ & $0.65681(11)$ & $0.0175(2)$ \\
H5A & 0.2725 & 0.7167 & 0.6361 & $0.021^{*}$
\end{tabular}




\begin{tabular}{|c|c|c|c|c|}
\hline C6 & $0.23518(14)$ & $0.47094(12)$ & $0.54616(11)$ & $0.0153(2)$ \\
\hline H6A & 0.1371 & 0.4957 & 0.5135 & $0.018^{*}$ \\
\hline $\mathrm{C} 7$ & $0.29303(14)$ & $0.35486(12)$ & $0.53359(10)$ & $0.0134(2)$ \\
\hline $\mathrm{C} 8$ & $0.20850(15)$ & $0.23836(12)$ & $0.46996(11)$ & $0.0155(2)$ \\
\hline $\mathrm{H} 8 \mathrm{~A}$ & 0.2831 & 0.1737 & 0.4649 & $0.019 *$ \\
\hline H8B & 0.1674 & 0.2574 & 0.3926 & $0.019^{*}$ \\
\hline C9 & $0.07088(14)$ & $0.19108(12)$ & $0.53330(11)$ & $0.0143(2)$ \\
\hline $\mathrm{C} 10$ & $0.05212(15)$ & $-0.02976(12)$ & $0.74143(11)$ & $0.0158(2)$ \\
\hline $\mathrm{H} 10 \mathrm{~A}$ & 0.1519 & -0.0582 & 0.7326 & $0.019 *$ \\
\hline C11 & $-0.03602(15)$ & $-0.08324(12)$ & $0.82756(11)$ & $0.0146(2)$ \\
\hline C12 & $0.03051(15)$ & $-0.17581(13)$ & $0.89465(12)$ & $0.0175(2)$ \\
\hline $\mathrm{H} 12 \mathrm{~A}$ & 0.1274 & -0.2048 & 0.8788 & $0.021 *$ \\
\hline $\mathrm{C} 13$ & $-0.04169(15)$ & $-0.22574(12)$ & $0.98328(11)$ & $0.0173(2)$ \\
\hline H13A & 0.0055 & -0.2883 & 1.0247 & $0.021 *$ \\
\hline C14 & $-0.18834(15)$ & $-0.18158(12)$ & $1.01136(10)$ & $0.0144(2)$ \\
\hline $\mathrm{C} 15$ & $-0.25764(15)$ & $-0.08989(12)$ & $0.94303(11)$ & $0.0156(2)$ \\
\hline $\mathrm{H} 15 \mathrm{~A}$ & -0.3549 & -0.0610 & 0.9582 & $0.019 *$ \\
\hline $\mathrm{C} 16$ & $-0.18382(15)$ & $-0.04205(12)$ & $0.85370(11)$ & $0.0147(2)$ \\
\hline $\mathrm{C} 17$ & $-0.19118(17)$ & $-0.32564(13)$ & $1.16815(12)$ & $0.0196(2)$ \\
\hline H17A & -0.2326 & -0.3243 & 1.2416 & $0.024 *$ \\
\hline H17B & -0.0760 & -0.3076 & 1.1831 & $0.024 *$ \\
\hline $\mathrm{C} 18$ & $-0.2262(2)$ & $-0.45760(14)$ & $1.11027(14)$ & $0.0265(3)$ \\
\hline $\mathrm{H} 18 \mathrm{~A}$ & -0.1691 & -0.5164 & 1.1555 & $0.040 *$ \\
\hline H18B & -0.1926 & -0.4586 & 1.0351 & $0.040 *$ \\
\hline $\mathrm{H} 18 \mathrm{C}$ & -0.3391 & -0.4808 & 1.1038 & $0.040 *$ \\
\hline C19 & $-0.41481(16)$ & $-0.18615(13)$ & $1.12605(11)$ & $0.0186(2)$ \\
\hline H19A & -0.4149 & -0.0958 & 1.1202 & $0.022 *$ \\
\hline H19B & -0.4286 & -0.2039 & 1.2045 & $0.022 *$ \\
\hline $\mathrm{C} 20$ & $-0.55683(17)$ & $-0.25000(16)$ & $1.04598(13)$ & $0.0252(3)$ \\
\hline $\mathrm{H} 20 \mathrm{~A}$ & -0.6542 & -0.2176 & 1.0652 & $0.038 *$ \\
\hline $\mathrm{H} 20 \mathrm{~B}$ & -0.5615 & -0.3391 & 1.0544 & $0.038^{*}$ \\
\hline $\mathrm{H} 20 \mathrm{C}$ & -0.5440 & -0.2334 & 0.9680 & $0.038^{*}$ \\
\hline $\mathrm{O} 1 \mathrm{~W}$ & $0.64543(12)$ & $0.15365(9)$ & $0.57170(9)$ & $0.01893(19)$ \\
\hline $\mathrm{O} 2 \mathrm{~W}$ & $0.60246(12)$ & $0.01353(10)$ & $0.36855(10)$ & $0.01989(19)$ \\
\hline H1N3 & $0.205(2)$ & $0.0710(17)$ & $0.6120(15)$ & $0.018(4)^{*}$ \\
\hline $\mathrm{H} 1 \mathrm{O} 2$ & $-0.209(3)$ & $0.069(3)$ & $0.737(2)$ & $0.058(8)^{*}$ \\
\hline H1W1 & $0.587(3)$ & $0.215(2)$ & $0.582(2)$ & $0.041(6)^{*}$ \\
\hline $\mathrm{H} 2 \mathrm{~W} 1$ & $0.742(3)$ & $0.181(2)$ & $0.5720(18)$ & $0.031(5)^{*}$ \\
\hline H1W2 & $0.518(3)$ & $-0.026(2)$ & $0.369(2)$ & $0.041(6)^{*}$ \\
\hline $\mathrm{H} 2 \mathrm{~W} 2$ & $0.608(2)$ & $0.060(2)$ & $0.4315(18)$ & $0.026(5)^{*}$ \\
\hline
\end{tabular}

Atomic displacement parameters $\left(\AA^{2}\right)$

\begin{tabular}{lllllll}
\hline & $U^{11}$ & $U^{22}$ & $U^{33}$ & $U^{12}$ & $U^{13}$ & $U^{23}$ \\
\hline Br1 & $0.01455(7)$ & $0.02522(8)$ & $0.02311(8)$ & $0.00475(5)$ & $-0.00432(5)$ & $-0.00016(5)$ \\
O1 & $0.0123(4)$ & $0.0243(5)$ & $0.0255(5)$ & $0.0016(3)$ & $0.0027(4)$ & $0.0083(4)$ \\
O2 & $0.0220(5)$ & $0.0254(5)$ & $0.0236(5)$ & $0.0075(4)$ & $0.0069(4)$ & $0.0106(4)$ \\
N1 & $0.0117(4)$ & $0.0156(5)$ & $0.0132(5)$ & $0.0004(4)$ & $0.0019(4)$ & $0.0016(4)$
\end{tabular}


supporting information

\begin{tabular}{lllllll}
$\mathrm{N} 2$ & $0.0121(4)$ & $0.0152(5)$ & $0.0133(5)$ & $0.0009(3)$ & $0.0018(4)$ & $0.0027(4)$ \\
$\mathrm{N} 3$ & $0.0107(4)$ & $0.0166(5)$ & $0.0165(5)$ & $-0.0009(4)$ & $0.0041(4)$ & $0.0021(4)$ \\
$\mathrm{N} 4$ & $0.0126(4)$ & $0.0178(5)$ & $0.0147(5)$ & $-0.0017(4)$ & $0.0037(4)$ & $0.0009(4)$ \\
$\mathrm{N} 5$ & $0.0165(5)$ & $0.0194(5)$ & $0.0136(5)$ & $0.0012(4)$ & $0.0030(4)$ & $0.0031(4)$ \\
C1 & $0.0115(5)$ & $0.0154(5)$ & $0.0115(5)$ & $0.0013(4)$ & $0.0022(4)$ & $0.0031(4)$ \\
C2 & $0.0130(5)$ & $0.0177(6)$ & $0.0131(5)$ & $0.0007(4)$ & $0.0007(4)$ & $0.0020(4)$ \\
C3 & $0.0179(6)$ & $0.0194(6)$ & $0.0138(5)$ & $-0.0021(4)$ & $0.0005(4)$ & $0.0001(4)$ \\
C4 & $0.0229(6)$ & $0.0151(5)$ & $0.0167(6)$ & $0.0017(4)$ & $0.0036(5)$ & $0.0013(4)$ \\
C5 & $0.0199(6)$ & $0.0156(6)$ & $0.0178(6)$ & $0.0040(4)$ & $0.0036(5)$ & $0.0036(4)$ \\
C6 & $0.0109(5)$ & $0.0197(6)$ & $0.0148(5)$ & $0.0000(4)$ & $-0.0001(4)$ & $0.0026(4)$ \\
C7 & $0.0118(5)$ & $0.0171(5)$ & $0.0115(5)$ & $-0.0009(4)$ & $0.0030(4)$ & $0.0024(4)$ \\
C8 & $0.0136(5)$ & $0.0188(6)$ & $0.0140(5)$ & $-0.0030(4)$ & $0.0039(4)$ & $0.0004(4)$ \\
C9 & $0.0122(5)$ & $0.0160(5)$ & $0.0141(5)$ & $-0.0030(4)$ & $0.0017(4)$ & $0.0001(4)$ \\
C10 & $0.0129(5)$ & $0.0173(6)$ & $0.0169(6)$ & $-0.0007(4)$ & $0.0024(4)$ & $0.0003(4)$ \\
C11 & $0.0131(5)$ & $0.0164(5)$ & $0.0139(5)$ & $-0.0013(4)$ & $0.0010(4)$ & $0.0009(4)$ \\
C12 & $0.0132(5)$ & $0.0200(6)$ & $0.0193(6)$ & $0.0014(4)$ & $0.0017(4)$ & $0.0029(5)$ \\
C13 & $0.0154(5)$ & $0.0187(6)$ & $0.0176(6)$ & $0.0012(4)$ & $0.0004(4)$ & $0.0041(4)$ \\
C14 & $0.0155(5)$ & $0.0152(5)$ & $0.0113(5)$ & $-0.0022(4)$ & $-0.0003(4)$ & $-0.0002(4)$ \\
C15 & $0.0145(5)$ & $0.0173(6)$ & $0.0152(6)$ & $0.0010(4)$ & $0.0023(4)$ & $0.0007(4)$ \\
C16 & $0.0144(5)$ & $0.0150(5)$ & $0.0144(5)$ & $-0.0004(4)$ & $0.0008(4)$ & $0.0007(4)$ \\
C17 & $0.0227(6)$ & $0.0207(6)$ & $0.0158(6)$ & $0.0017(5)$ & $0.0024(5)$ & $0.0040(5)$ \\
C18 & $0.0312(8)$ & $0.0201(7)$ & $0.0288(8)$ & $0.0007(5)$ & $0.0058(6)$ & $0.0029(5)$ \\
C19 & $0.0193(6)$ & $0.0230(6)$ & $0.0145(6)$ & $0.0023(5)$ & $0.0055(5)$ & $0.0016(5)$ \\
C20 & $0.0177(6)$ & $0.0342(8)$ & $0.0235(7)$ & $-0.0006(5)$ & $0.0040(5)$ & $-0.0005(6)$ \\
O1W & $0.0137(4)$ & $0.0173(4)$ & $0.0263(5)$ & $0.0007(3)$ & $0.0043(4)$ & $0.0026(4)$ \\
O2W & $0.0150(4)$ & $0.0182(4)$ & $0.0270(5)$ & $0.0005(3)$ & $0.0057(4)$ & $0.0000(4)$ \\
& & & & & & \\
\hline
\end{tabular}

Geometric parameters $\left(\AA,{ }^{o}\right)$

\begin{tabular}{llll}
\hline $\mathrm{Br} 1-\mathrm{C} 2$ & $1.8809(13)$ & $\mathrm{C} 8-\mathrm{H} 8 \mathrm{~B}$ & 0.9700 \\
$\mathrm{O} 1-\mathrm{C} 9$ & $1.2354(16)$ & $\mathrm{C} 10-\mathrm{C} 11$ & $1.4444(17)$ \\
$\mathrm{O} 2-\mathrm{C} 16$ & $1.3534(16)$ & $\mathrm{C} 10-\mathrm{H} 10 \mathrm{~A}$ & 0.9300 \\
$\mathrm{O} 2-\mathrm{H} 1 \mathrm{O} 2$ & $0.87(3)$ & $\mathrm{C} 11-\mathrm{C} 12$ & $1.4007(18)$ \\
$\mathrm{N} 1-\mathrm{C} 1$ & $1.3350(16)$ & $\mathrm{C} 11-\mathrm{C} 16$ & $1.4134(17)$ \\
$\mathrm{N} 1-\mathrm{C} 7$ & $1.3804(16)$ & $\mathrm{C} 12-\mathrm{C} 13$ & $1.3804(18)$ \\
$\mathrm{N} 2-\mathrm{C} 5$ & $1.3802(16)$ & $\mathrm{C} 12-\mathrm{H} 12 \mathrm{~A}$ & 0.9300 \\
$\mathrm{~N} 2-\mathrm{C} 6$ & $1.3837(16)$ & $\mathrm{C} 13-\mathrm{C} 14$ & $1.4232(18)$ \\
$\mathrm{N} 2-\mathrm{C} 1$ & $1.3887(15)$ & $\mathrm{C} 13-\mathrm{H} 13 \mathrm{~A}$ & 0.9300 \\
$\mathrm{~N} 3-\mathrm{C} 9$ & $1.3443(17)$ & $\mathrm{C} 14-\mathrm{C} 15$ & $1.4108(17)$ \\
$\mathrm{N} 3-\mathrm{N} 4$ & $1.3906(14)$ & $\mathrm{C} 15-\mathrm{C} 16$ & $1.3856(18)$ \\
$\mathrm{N} 3-\mathrm{H} 1 \mathrm{~N} 3$ & $0.896(19)$ & $\mathrm{C} 15-\mathrm{H} 15 \mathrm{~A}$ & 0.9300 \\
$\mathrm{~N} 4-\mathrm{C} 10$ & $1.2915(17)$ & $\mathrm{C} 17-\mathrm{C} 18$ & $1.526(2)$ \\
$\mathrm{N} 5-\mathrm{C} 14$ & $1.3690(16)$ & $\mathrm{C} 17-\mathrm{H} 17 \mathrm{~A}$ & 0.9700 \\
$\mathrm{~N} 5-\mathrm{C} 17$ & $1.4594(17)$ & $\mathrm{C} 17-\mathrm{H} 17 \mathrm{~B}$ & 0.9700 \\
$\mathrm{~N} 5-\mathrm{C} 19$ & $1.4642(17)$ & $\mathrm{C} 18-\mathrm{H} 18 \mathrm{~A}$ & 0.9600 \\
$\mathrm{C} 1-\mathrm{C} 2$ & $1.4170(17)$ & $\mathrm{C} 18-\mathrm{H} 18 \mathrm{~B}$ & 0.9600 \\
$\mathrm{C} 2-\mathrm{C} 3$ & $1.3662(18)$ & $\mathrm{C} 18-\mathrm{H} 18 \mathrm{C}$ & 0.9600 \\
$\mathrm{C} 3-\mathrm{C} 4$ & $1.4216(19)$ & $\mathrm{C} 19-\mathrm{C} 20$ & $1.528(2)$
\end{tabular}




\begin{tabular}{|c|c|c|c|}
\hline $\mathrm{C} 3-\mathrm{H} 3 \mathrm{~A}$ & 0.9300 & C19-H19A & 0.9700 \\
\hline $\mathrm{C} 4-\mathrm{C} 5$ & $1.3548(19)$ & C19-H19B & 0.9700 \\
\hline $\mathrm{C} 4-\mathrm{H} 4 \mathrm{~A}$ & 0.9300 & $\mathrm{C} 20-\mathrm{H} 20 \mathrm{~A}$ & 0.9600 \\
\hline $\mathrm{C} 5-\mathrm{H} 5 \mathrm{~A}$ & 0.9300 & $\mathrm{C} 20-\mathrm{H} 20 \mathrm{~B}$ & 0.9600 \\
\hline $\mathrm{C} 6-\mathrm{C} 7$ & $1.3701(17)$ & $\mathrm{C} 20-\mathrm{H} 20 \mathrm{C}$ & 0.9600 \\
\hline C6-H6A & 0.9300 & $\mathrm{O} 1 \mathrm{~W}-\mathrm{H} 1 \mathrm{~W} 1$ & $0.86(3)$ \\
\hline $\mathrm{C} 7-\mathrm{C} 8$ & $1.5057(17)$ & $\mathrm{O} 1 \mathrm{~W}-\mathrm{H} 2 \mathrm{~W} 1$ & $0.84(2)$ \\
\hline $\mathrm{C} 8-\mathrm{C} 9$ & $1.5242(17)$ & $\mathrm{O} 2 \mathrm{~W}-\mathrm{H} 1 \mathrm{~W} 2$ & $0.80(3)$ \\
\hline $\mathrm{C} 8-\mathrm{H} 8 \mathrm{~A}$ & 0.9700 & $\mathrm{O} 2 \mathrm{~W}-\mathrm{H} 2 \mathrm{~W} 2$ & $0.86(2)$ \\
\hline $\mathrm{C} 16-\mathrm{O} 2-\mathrm{H} 1 \mathrm{O} 2$ & $111.7(18)$ & $\mathrm{C} 12-\mathrm{C} 11-\mathrm{C} 16$ & $116.99(11)$ \\
\hline $\mathrm{C} 1-\mathrm{N} 1-\mathrm{C} 7$ & $105.16(10)$ & $\mathrm{C} 12-\mathrm{C} 11-\mathrm{C} 10$ & $119.37(11)$ \\
\hline $\mathrm{C} 5-\mathrm{N} 2-\mathrm{C} 6$ & $130.13(11)$ & $\mathrm{C} 16-\mathrm{C} 11-\mathrm{C} 10$ & $123.53(12)$ \\
\hline $\mathrm{C} 5-\mathrm{N} 2-\mathrm{C} 1$ & $123.09(11)$ & $\mathrm{C} 13-\mathrm{C} 12-\mathrm{C} 11$ & $122.76(12)$ \\
\hline $\mathrm{C} 6-\mathrm{N} 2-\mathrm{C} 1$ & $106.77(10)$ & $\mathrm{C} 13-\mathrm{C} 12-\mathrm{H} 12 \mathrm{~A}$ & 118.6 \\
\hline $\mathrm{C} 9-\mathrm{N} 3-\mathrm{N} 4$ & $120.60(10)$ & $\mathrm{C} 11-\mathrm{C} 12-\mathrm{H} 12 \mathrm{~A}$ & 118.6 \\
\hline $\mathrm{C} 9-\mathrm{N} 3-\mathrm{H} 1 \mathrm{~N} 3$ & $121.0(12)$ & $\mathrm{C} 12-\mathrm{C} 13-\mathrm{C} 14$ & 120.09 (12) \\
\hline $\mathrm{N} 4-\mathrm{N} 3-\mathrm{H} 1 \mathrm{~N} 3$ & $118.2(12)$ & $\mathrm{C} 12-\mathrm{C} 13-\mathrm{H} 13 \mathrm{~A}$ & 120.0 \\
\hline $\mathrm{C} 10-\mathrm{N} 4-\mathrm{N} 3$ & $113.55(10)$ & $\mathrm{C} 14-\mathrm{C} 13-\mathrm{H} 13 \mathrm{~A}$ & 120.0 \\
\hline $\mathrm{C} 14-\mathrm{N} 5-\mathrm{C} 17$ & $120.87(11)$ & $\mathrm{N} 5-\mathrm{C} 14-\mathrm{C} 15$ & $121.23(11)$ \\
\hline $\mathrm{C} 14-\mathrm{N} 5-\mathrm{C} 19$ & $121.09(11)$ & $\mathrm{N} 5-\mathrm{C} 14-\mathrm{C} 13$ & $121.24(12)$ \\
\hline $\mathrm{C} 17-\mathrm{N} 5-\mathrm{C} 19$ & $117.68(11)$ & $\mathrm{C} 15-\mathrm{C} 14-\mathrm{C} 13$ & $117.54(11)$ \\
\hline $\mathrm{N} 1-\mathrm{C} 1-\mathrm{N} 2$ & $111.09(10)$ & $\mathrm{C} 16-\mathrm{C} 15-\mathrm{C} 14$ & $121.34(12)$ \\
\hline $\mathrm{N} 1-\mathrm{C} 1-\mathrm{C} 2$ & $131.60(11)$ & $\mathrm{C} 16-\mathrm{C} 15-\mathrm{H} 15 \mathrm{~A}$ & 119.3 \\
\hline $\mathrm{N} 2-\mathrm{C} 1-\mathrm{C} 2$ & $117.31(11)$ & $\mathrm{C} 14-\mathrm{C} 15-\mathrm{H} 15 \mathrm{~A}$ & 119.3 \\
\hline $\mathrm{C} 3-\mathrm{C} 2-\mathrm{C} 1$ & $120.47(12)$ & $\mathrm{O} 2-\mathrm{C} 16-\mathrm{C} 15$ & $116.85(11)$ \\
\hline $\mathrm{C} 3-\mathrm{C} 2-\mathrm{Br} 1$ & $121.18(10)$ & $\mathrm{O} 2-\mathrm{C} 16-\mathrm{C} 11$ & $121.89(12)$ \\
\hline $\mathrm{C} 1-\mathrm{C} 2-\mathrm{Br} 1$ & $118.31(9)$ & $\mathrm{C} 15-\mathrm{C} 16-\mathrm{C} 11$ & $121.25(12)$ \\
\hline $\mathrm{C} 2-\mathrm{C} 3-\mathrm{C} 4$ & $119.53(12)$ & $\mathrm{N} 5-\mathrm{C} 17-\mathrm{C} 18$ & $114.42(12)$ \\
\hline $\mathrm{C} 2-\mathrm{C} 3-\mathrm{H} 3 \mathrm{~A}$ & 120.2 & N5-C17-H17A & 108.7 \\
\hline $\mathrm{C} 4-\mathrm{C} 3-\mathrm{H} 3 \mathrm{~A}$ & 120.2 & $\mathrm{C} 18-\mathrm{C} 17-\mathrm{H} 17 \mathrm{~A}$ & 108.7 \\
\hline $\mathrm{C} 5-\mathrm{C} 4-\mathrm{C} 3$ & $121.06(12)$ & N5-C17-H17B & 108.7 \\
\hline $\mathrm{C} 5-\mathrm{C} 4-\mathrm{H} 4 \mathrm{~A}$ & 119.5 & $\mathrm{C} 18-\mathrm{C} 17-\mathrm{H} 17 \mathrm{~B}$ & 108.7 \\
\hline $\mathrm{C} 3-\mathrm{C} 4-\mathrm{H} 4 \mathrm{~A}$ & 119.5 & $\mathrm{H} 17 \mathrm{~A}-\mathrm{C} 17-\mathrm{H} 17 \mathrm{~B}$ & 107.6 \\
\hline $\mathrm{C} 4-\mathrm{C} 5-\mathrm{N} 2$ & $118.52(12)$ & $\mathrm{C} 17-\mathrm{C} 18-\mathrm{H} 18 \mathrm{~A}$ & 109.5 \\
\hline $\mathrm{C} 4-\mathrm{C} 5-\mathrm{H} 5 \mathrm{~A}$ & 120.7 & $\mathrm{C} 17-\mathrm{C} 18-\mathrm{H} 18 \mathrm{~B}$ & 109.5 \\
\hline $\mathrm{N} 2-\mathrm{C} 5-\mathrm{H} 5 \mathrm{~A}$ & 120.7 & $\mathrm{H} 18 \mathrm{~A}-\mathrm{C} 18-\mathrm{H} 18 \mathrm{~B}$ & 109.5 \\
\hline $\mathrm{C} 7-\mathrm{C} 6-\mathrm{N} 2$ & $105.77(11)$ & $\mathrm{C} 17-\mathrm{C} 18-\mathrm{H} 18 \mathrm{C}$ & 109.5 \\
\hline C $7-\mathrm{C} 6-\mathrm{H} 6 \mathrm{~A}$ & 127.1 & $\mathrm{H} 18 \mathrm{~A}-\mathrm{C} 18-\mathrm{H} 18 \mathrm{C}$ & 109.5 \\
\hline $\mathrm{N} 2-\mathrm{C} 6-\mathrm{H} 6 \mathrm{~A}$ & 127.1 & $\mathrm{H} 18 \mathrm{~B}-\mathrm{C} 18-\mathrm{H} 18 \mathrm{C}$ & 109.5 \\
\hline $\mathrm{C} 6-\mathrm{C} 7-\mathrm{N} 1$ & $111.22(11)$ & $\mathrm{N} 5-\mathrm{C} 19-\mathrm{C} 20$ & $113.89(11)$ \\
\hline $\mathrm{C} 6-\mathrm{C} 7-\mathrm{C} 8$ & $127.58(11)$ & N5-C19-H19A & 108.8 \\
\hline $\mathrm{N} 1-\mathrm{C} 7-\mathrm{C} 8$ & $121.11(11)$ & $\mathrm{C} 20-\mathrm{C} 19-\mathrm{H} 19 \mathrm{~A}$ & 108.8 \\
\hline $\mathrm{C} 7-\mathrm{C} 8-\mathrm{C} 9$ & $109.35(10)$ & N5-C19-H19B & 108.8 \\
\hline $\mathrm{C} 7-\mathrm{C} 8-\mathrm{H} 8 \mathrm{~A}$ & 109.8 & $\mathrm{C} 20-\mathrm{C} 19-\mathrm{H} 19 \mathrm{~B}$ & 108.8 \\
\hline $\mathrm{C} 9-\mathrm{C} 8-\mathrm{H} 8 \mathrm{~A}$ & 109.8 & H19A-C19-H19B & 107.7 \\
\hline $\mathrm{C} 7-\mathrm{C} 8-\mathrm{H} 8 \mathrm{~B}$ & 109.8 & $\mathrm{C} 19-\mathrm{C} 20-\mathrm{H} 20 \mathrm{~A}$ & 109.5 \\
\hline $\mathrm{C} 9-\mathrm{C} 8-\mathrm{H} 8 \mathrm{~B}$ & 109.8 & $\mathrm{C} 19-\mathrm{C} 20-\mathrm{H} 20 \mathrm{~B}$ & 109.5 \\
\hline
\end{tabular}




\begin{tabular}{|c|c|}
\hline $\mathrm{H} 8 \mathrm{~A}-\mathrm{C} 8-\mathrm{H} 8 \mathrm{~B}$ & 108.3 \\
\hline $\mathrm{O} 1-\mathrm{C} 9-\mathrm{N} 3$ & $124.94(11)$ \\
\hline $\mathrm{O} 1-\mathrm{C} 9-\mathrm{C} 8$ & $120.90(12)$ \\
\hline $\mathrm{N} 3-\mathrm{C} 9-\mathrm{C} 8$ & $114.12(11)$ \\
\hline $\mathrm{N} 4-\mathrm{C} 10-\mathrm{C} 11$ & $123.10(12)$ \\
\hline N4-C10-H10A & 118.5 \\
\hline $\mathrm{C} 11-\mathrm{C} 10-\mathrm{H} 10 \mathrm{~A}$ & 118.5 \\
\hline $\mathrm{C} 9-\mathrm{N} 3-\mathrm{N} 4-\mathrm{C} 10$ & $-178.57(11)$ \\
\hline $\mathrm{C} 7-\mathrm{N} 1-\mathrm{C} 1-\mathrm{N} 2$ & $0.28(13)$ \\
\hline $\mathrm{C} 7-\mathrm{N} 1-\mathrm{C} 1-\mathrm{C} 2$ & $-179.63(13)$ \\
\hline $\mathrm{C} 5-\mathrm{N} 2-\mathrm{C} 1-\mathrm{N} 1$ & $-179.26(11)$ \\
\hline $\mathrm{C} 6-\mathrm{N} 2-\mathrm{C} 1-\mathrm{N} 1$ & $-0.44(13)$ \\
\hline $\mathrm{C} 5-\mathrm{N} 2-\mathrm{C} 1-\mathrm{C} 2$ & $0.66(17)$ \\
\hline $\mathrm{C} 6-\mathrm{N} 2-\mathrm{C} 1-\mathrm{C} 2$ & $179.48(11)$ \\
\hline $\mathrm{N} 1-\mathrm{C} 1-\mathrm{C} 2-\mathrm{C} 3$ & $-179.62(13)$ \\
\hline $\mathrm{N} 2-\mathrm{C} 1-\mathrm{C} 2-\mathrm{C} 3$ & $0.47(18)$ \\
\hline $\mathrm{N} 1-\mathrm{C} 1-\mathrm{C} 2-\mathrm{Br} 1$ & $2.65(19)$ \\
\hline $\mathrm{N} 2-\mathrm{C} 1-\mathrm{C} 2-\mathrm{Br} 1$ & $-177.26(8)$ \\
\hline $\mathrm{C} 1-\mathrm{C} 2-\mathrm{C} 3-\mathrm{C} 4$ & $-1.02(19)$ \\
\hline $\mathrm{Br} 1-\mathrm{C} 2-\mathrm{C} 3-\mathrm{C} 4$ & $176.64(10)$ \\
\hline $\mathrm{C} 2-\mathrm{C} 3-\mathrm{C} 4-\mathrm{C} 5$ & $0.5(2)$ \\
\hline $\mathrm{C} 3-\mathrm{C} 4-\mathrm{C} 5-\mathrm{N} 2$ & $0.6(2)$ \\
\hline $\mathrm{C} 6-\mathrm{N} 2-\mathrm{C} 5-\mathrm{C} 4$ & $-179.72(12)$ \\
\hline $\mathrm{C} 1-\mathrm{N} 2-\mathrm{C} 5-\mathrm{C} 4$ & $-1.20(19)$ \\
\hline $\mathrm{C} 5-\mathrm{N} 2-\mathrm{C} 6-\mathrm{C} 7$ & $179.12(12)$ \\
\hline $\mathrm{C} 1-\mathrm{N} 2-\mathrm{C} 6-\mathrm{C} 7$ & $0.41(13)$ \\
\hline $\mathrm{N} 2-\mathrm{C} 6-\mathrm{C} 7-\mathrm{N} 1$ & $-0.25(14)$ \\
\hline $\mathrm{N} 2-\mathrm{C} 6-\mathrm{C} 7-\mathrm{C} 8$ & $176.21(11)$ \\
\hline $\mathrm{C} 1-\mathrm{N} 1-\mathrm{C} 7-\mathrm{C} 6$ & $-0.01(14)$ \\
\hline $\mathrm{C} 1-\mathrm{N} 1-\mathrm{C} 7-\mathrm{C} 8$ & $-176.73(11)$ \\
\hline $\mathrm{C} 6-\mathrm{C} 7-\mathrm{C} 8-\mathrm{C} 9$ & $-68.28(16)$ \\
\hline $\mathrm{N} 1-\mathrm{C} 7-\mathrm{C} 8-\mathrm{C} 9$ & $107.87(13)$ \\
\hline $\mathrm{N} 4-\mathrm{N} 3-\mathrm{C} 9-\mathrm{O} 1$ & $-3.51(19)$ \\
\hline $\mathrm{N} 4-\mathrm{N} 3-\mathrm{C} 9-\mathrm{C} 8$ & $174.06(10)$ \\
\hline
\end{tabular}

$\mathrm{H} 20 \mathrm{~A}-\mathrm{C} 20-\mathrm{H} 20 \mathrm{~B}$

$\mathrm{C} 19-\mathrm{C} 20-\mathrm{H} 20 \mathrm{C}$

$\mathrm{H} 20 \mathrm{~A}-\mathrm{C} 20-\mathrm{H} 20 \mathrm{C}$

$\mathrm{H} 20 \mathrm{~B}-\mathrm{C} 20-\mathrm{H} 20 \mathrm{C}$

$\mathrm{H} 1 \mathrm{~W} 1-\mathrm{O} 1 \mathrm{~W}-\mathrm{H} 2 \mathrm{~W} 1$

$\mathrm{H} 1 \mathrm{~W} 2-\mathrm{O} 2 \mathrm{~W}-\mathrm{H} 2 \mathrm{~W} 2$

$\mathrm{C} 7-\mathrm{C} 8-\mathrm{C} 9-\mathrm{O} 1$

$\mathrm{C} 7-\mathrm{C} 8-\mathrm{C} 9-\mathrm{N} 3$

N3-N4-C10-C11

$\mathrm{N} 4-\mathrm{C} 10-\mathrm{C} 11-\mathrm{C} 12$

$\mathrm{N} 4-\mathrm{C} 10-\mathrm{C} 11-\mathrm{C} 16$

$\mathrm{C} 16-\mathrm{C} 11-\mathrm{C} 12-\mathrm{C} 13$

$\mathrm{C} 10-\mathrm{C} 11-\mathrm{C} 12-\mathrm{C} 13$

$\mathrm{C} 11-\mathrm{C} 12-\mathrm{C} 13-\mathrm{C} 14$

C17-N5-C14-C15

C19-N5-C14-C15

C17-N5-C14-C13

C19-N5-C14-C13

C12-C13-C14-N5

$\mathrm{C} 12-\mathrm{C} 13-\mathrm{C} 14-\mathrm{C} 15$

$\mathrm{N} 5-\mathrm{C} 14-\mathrm{C} 15-\mathrm{C} 16$

$\mathrm{C} 13-\mathrm{C} 14-\mathrm{C} 15-\mathrm{C} 16$

$\mathrm{C} 14-\mathrm{C} 15-\mathrm{C} 16-\mathrm{O} 2$

$\mathrm{C} 14-\mathrm{C} 15-\mathrm{C} 16-\mathrm{C} 11$

$\mathrm{C} 12-\mathrm{C} 11-\mathrm{C} 16-\mathrm{O} 2$

$\mathrm{C} 10-\mathrm{C} 11-\mathrm{C} 16-\mathrm{O} 2$

$\mathrm{C} 12-\mathrm{C} 11-\mathrm{C} 16-\mathrm{C} 15$

$\mathrm{C} 10-\mathrm{C} 11-\mathrm{C} 16-\mathrm{C} 15$

C14-N5-C17-C18

C19-N5-C17-C18

$\mathrm{C} 14-\mathrm{N} 5-\mathrm{C} 19-\mathrm{C} 20$

C17-N5-C19-C20
109.5

109.5

109.5

109.5

110 (2)

$101(2)$

$81.70(15)$

$-95.99(13)$

$175.89(11)$

$179.87(12)$

$-4.1(2)$

$-0.39(19)$

$175.88(12)$

$-1.3(2)$

176.84 (12)

3.84 (18)

$-3.34(18)$

$-176.34(12)$

-177.55 (12)

2.28 (19)

178.18 (12)

$-1.64(18)$

$179.42(11)$

-0.03 (19)

-178.35 (12)

5.54 (19)

1.06 (18)

$-175.04(12)$

-78.07 (16)

$95.16(14)$

77.22 (16)

-96.00 (14)

Hydrogen-bond geometry $\left(\hat{A},{ }^{\circ}\right)$

$\mathrm{Cg} 1$ is the centroid of the $\mathrm{N} 1 / \mathrm{C} 1 / \mathrm{N} 2 / \mathrm{C} 6 / \mathrm{C} 7$ ring and $\mathrm{Cg} 3$ is the centroid of the $\mathrm{C} 11-\mathrm{C} 16$ ring.

\begin{tabular}{lllll}
\hline$D-\mathrm{H} \cdots A$ & $D-\mathrm{H}$ & $\mathrm{H} \cdots A$ & $D \cdots A$ & $D-\mathrm{H}^{\cdots} A$ \\
\hline $\mathrm{N} 3-\mathrm{H} 1 N 3 \cdots \mathrm{O} 2 W^{\mathrm{i}}$ & $0.894(17)$ & $1.908(17)$ & $2.7956(15)$ & $171.7(17)$ \\
$\mathrm{O} 2-\mathrm{H} 1 O 2 \cdots \mathrm{O} 1 W^{\mathrm{ii}}$ & $0.87(3)$ & $2.42(3)$ & $2.9423(15)$ & $119(2)$ \\
$\mathrm{O} 2-\mathrm{H} 1 O 2 \cdots \mathrm{N} 4$ & $0.87(3)$ & $1.99(3)$ & $2.7181(16)$ & $142(2)$ \\
$\mathrm{O} 1 W-\mathrm{H} 1 W 1 \cdots \mathrm{N} 1$ & $0.86(2)$ & $1.98(2)$ & $2.8315(14)$ & $176(2)$ \\
$\mathrm{O} 1 W-\mathrm{H} 2 W \cdots \mathrm{O} 1{ }^{\mathrm{iii}}$ & $0.85(3)$ & $1.92(2)$ & $2.7361(14)$ & $162(2)$ \\
$\mathrm{O} 2 W-\mathrm{H} 1 W 2 \cdots \mathrm{O} 1 W^{\mathrm{i}}$ & $0.80(2)$ & $2.08(2)$ & $2.8311(15)$ & $157(2)$ \\
$\mathrm{O} 2 W-\mathrm{H} 2 W 2 \cdots \mathrm{O} 1 W$ & $0.86(2)$ & $1.87(2)$ & $2.7245(15)$ & $172.9(17)$ \\
$\mathrm{C} 5-\mathrm{H} 5 A \cdots \mathrm{O} 1^{\mathrm{iv}}$ & 0.93 & 2.50 & $3.3121(17)$ & 146
\end{tabular}


supporting information

\begin{tabular}{lllll}
$\mathrm{C} 10-\mathrm{H} 10 A \cdots \mathrm{O} 2 W^{\mathrm{i}}$ & 0.93 & 2.54 & $3.3256(17)$ & 142 \\
$\mathrm{C} 17-\mathrm{H} 17 B \cdots B^{\mathrm{v}} 1^{\mathrm{v}}$ & 0.97 & 2.85 & $3.6569(15)$ & 142 \\
$\mathrm{C} 3-\mathrm{H} 3 A \cdots C g 3^{\mathrm{vi}}$ & 0.93 & 2.61 & $3.4734(15)$ & 154 \\
$\mathrm{C} 17-\mathrm{H} 17 A \cdots C g 1^{1 \mathrm{vi}}$ & 0.97 & 2.70 & $3.5863(15)$ & 152 \\
\hline
\end{tabular}

Symmetry codes: (i) $-x+1,-y,-z+1$; (ii) $x-1, y, z$; (iii) $x+1, y, z$; (iv) $-x,-y+1,-z+1$; (v) $-x+1,-y,-z+2$; (vi) $x+1, y+1, z$; (vii) $-x,-y,-z+2$. 